\title{
XWAKE 1.1: A NEW IMPEDANCE AND WAKE FIELD SOFTWARE PACKAGE
}

\author{
G. W. Saewert and T. G. Jurgens, Fermi National Accelerator Laboratory, Batavia, IL 60510 USA
}

\begin{abstract}
This paper outlines the novel features found in Xwake 1.1. This release of Xwake is a body of revolution (BOR) conformal finite difference time domain (FDTD) code written to model longitudinal and transverse wake fields and impedances. The package is capable of accurately modeling slowly tapered structures as well as devices containing dielectric and permeable media. A state of the art perfectly matched layer (PML) absorbing boundary condition $(\mathrm{ABC})$ provides the ability to truncate the computational grid immediately at a devices' beam pipe openings, increasing the computational efficiency of the package. The program includes automatic mesh generator, field solver and post processor plotting modules all integrated with an intuitive graphical user interface. Since the package is written with ANSI-C and the Motif widget set, Xwake 1.1 is portable across UNIX environments. The use of dynamic memory allocation enables the program to automatically scale itself to the problem size without resorting to code recompilation.
\end{abstract}

\section{INTRODUCTION}

Xwake is a computer code that computes the impedance of particle accelerator beam line structures. As with present codes, this is accomplished by driving a body of revolution finite difference electromagnetic field solver [1] with a rigid relativistically moving particle bunch. The fields produced by the bunch are integrated to get wake potential and impedance. However Xwake overcomes limitations of current codes.

Xwake includes numerous features to make it easy to use. Its graphical user interface (GUI) was designed to be intuitive and is built with the popular X/Motif widgets. This along with strict adherence to ANSI-C give it portability across UNIX platforms. Xwake allocates the memory it needs dynamically at run time, thus the problem size it can handle is limited only by the installed computer memory.

\section{XWAKE 1.1 FEATURES}

The upcoming summer 19951.1 release of Xwake incorporates the following advanced features: the ability to model permeable, dielectric and lossy material, conformal modeling of an object's shape, and a state of the art broadband absorbing boundary condition (ABC). In addition, Xwake 1.1 can perform both monopole and dipole calculations, whereas Xwake 1.0 did monopole calculations only.

Xwake can model materially inhomogeneous objects with arbitrary permeability, permittivity and conductivity. Codes commonly used in accelerator physics such as ABCI [2] and TBCI [3] are limited in their ability to represent the material composition. The only material other than a vacuum they can model is a perfect electrical conductor.

Xwake deforms the shape of computational cells so that they conform to and therefore preserve the shape of the material in- terface [4]. Only cells immediately adjacent to the interface are deformed from the original rectangular cell shape. This means that the cost of utilizing the contour FDTD method over conventional FDTD methods is asymptotically negligible. Traditionally used codes use either a rectangular cell approximation (TBCI), or a diagonal unit cell approximation (ABCI) to describe the structure's shape. This level of geometrical accuracy is sufficient for the efficient computation of some structures, particularly when the media interfaces coincide with computuational cell boundaries. However, previously reported results from Xwake 1.0 [5], showed a 64-fold savings in computer memory and a 512-fold savings in computer run time over ABCI to obtain equal accuracy in the modeling of a slowly tapered beam pipe.

The Xwake $\mathrm{ABC}$ is an extension [6] of Berenger's PML ABC. The ABC reflection coefficient is routinely below $-80 \mathrm{db}$ for arbitrary wave angles and a broad range of frequencies. This means multiple beam pipe modes will be simultaneously absorbed, thus lowering the computational lattice noise due to spurious mode reflections. This contrasts with the (single) mode matching technique used in other codes. Additionally this means that the beam pipe can be truncated closer to the region of interest. This decreases the use of computer resources.

To use the program first describe the problem in a text file, then run Xwake to input the text file and subsequently obtain solutions. Options for executing the mesh generator, solver/post processor and plot creator are provided by the controls of the GUI, enabling the user to make repeated calculations at will with different parameters without leaving the program. The user meshes the problem with either a stepped edge or contour approximation. Wake potentials and impedances are calculated by the solver using six user adjustable parameters with default values predetermined for convenience, likewise with the plotting options. Plots can be saved to a PostScript file or sent directly to a PostScript printer.

\section{RESULTS}

This section presents modeling results produces by Xwake 1.1. The examples chosen illustrate the salient features of the code.

\section{A. Radial Transmission Line}

The first structure modeled is a shorted radial transmission line. Both an empty and a ferrite loaded version are investigated. Figure 1 illustrates the problem at hand. The transmission line is $5 \mathrm{~cm}$ wide with a depth of $20 \mathrm{~cm}$. The ferrite occupies the upper $15 \mathrm{~cm}$ of the transmission line. The beam pipe radius is $10 \mathrm{~cm}$. The radial and longitudinal discretization lengths are $5 \mathrm{~mm}$. The (hollow) beam radius coincides with the beam pipe and has an rms length of $2 \mathrm{~cm}$. Figure 2 displays the monopole longitudinal impedance of the empty transmission line. An equivalent ABCI simulation provides similar results. Figures 3 and 4 show the dipole longitudinal and transverse wake potentials of the empty transmission line. Figure 5 is a plot of the dipole wake potentials 
Ferrite Loaded Radial Transmission Line (mu=10) XWAKE 1.1 Thu Apr 27 10:12:09 1995

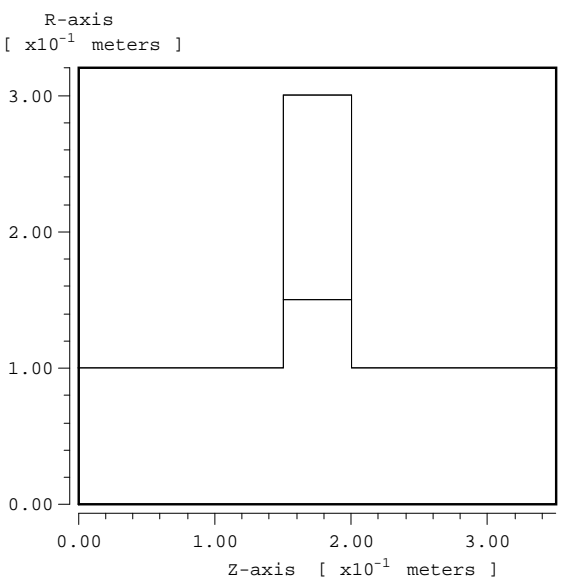

Figure. 1. Radial Transmission Line Geometry

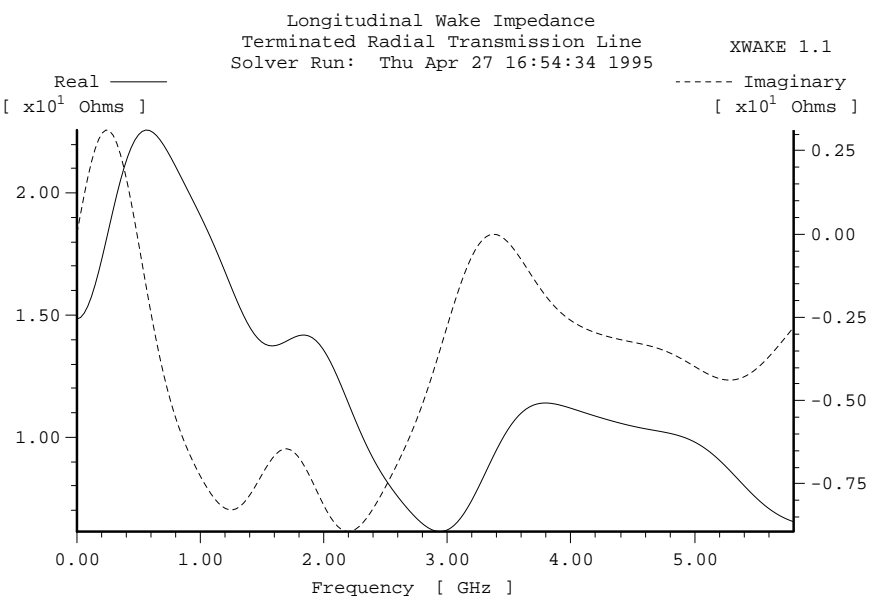

Figure. 2. Monopole Longitudinal Impedance

calculated by ABCI version 8.8 for the same geometry, beam parameters and grid discretization lengths as modeled with Xwake. The Xwake and $\mathrm{ABCI}$ results are virtually identical. Note that the media interfaces coincide with the rectangular computational cell boundaries, which assures an accurate shape representation without deforming the cells.

Figure 6 is a graph of the monopole longitudinal impedance of the radial transmission line, this time loaded with a ferrite with a relative permeability of $\mu_{r}=10$.

\section{B. Beam Line Bellows}

This example illustrates the advantage gained in using a contour geometry approximation over using a stepped approximation. Figure 7 depicts a ten convolution beam line bellow with outer and inner radii of $3.8 \mathrm{~cm}$ and $2.7 \mathrm{~cm}$. The beam and beam pipe radii are also $2.7 \mathrm{~cm}$. The radial and longitudinal discretization lengths are $0.5 \mathrm{~mm}$ and the beam rms length is $1 \mathrm{~cm}$. Figure 8 shows a comparison of the Xwake computed monopole longitudinal wake potential using the contour cell and the rectangular cell (stepped) approximations. The contour FDTD model has converged and that is the contour data set plotted. The stepped FDTD data shown has a discretization of $0.5 \mathrm{~mm}$, however it has

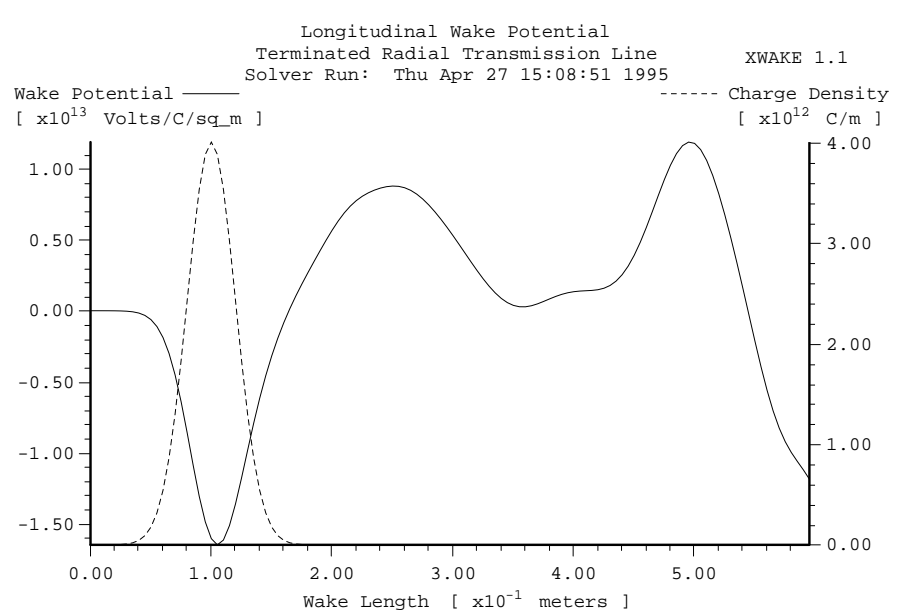

Figure. 3. Dipole Longitudinal Wake Potential

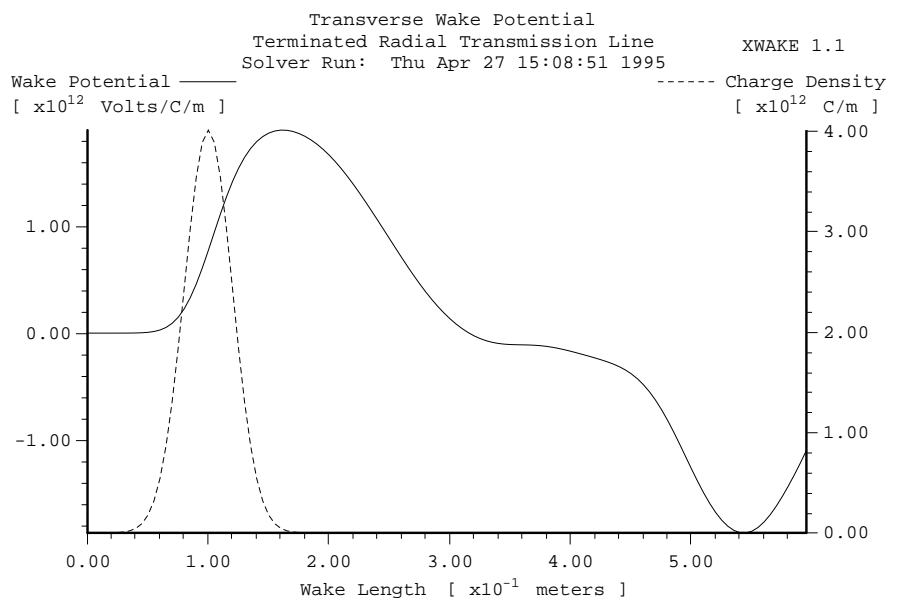

Figure. 4. Dipole Transverse Wake Potential

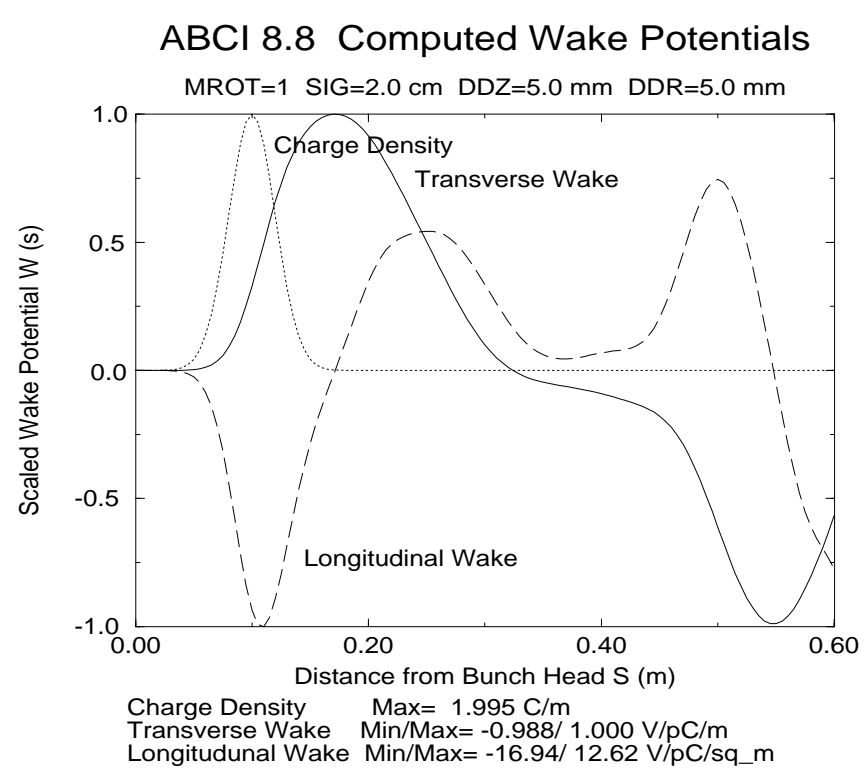

Figure. 5. ABCI Calculated Dipole Wake Potentials 


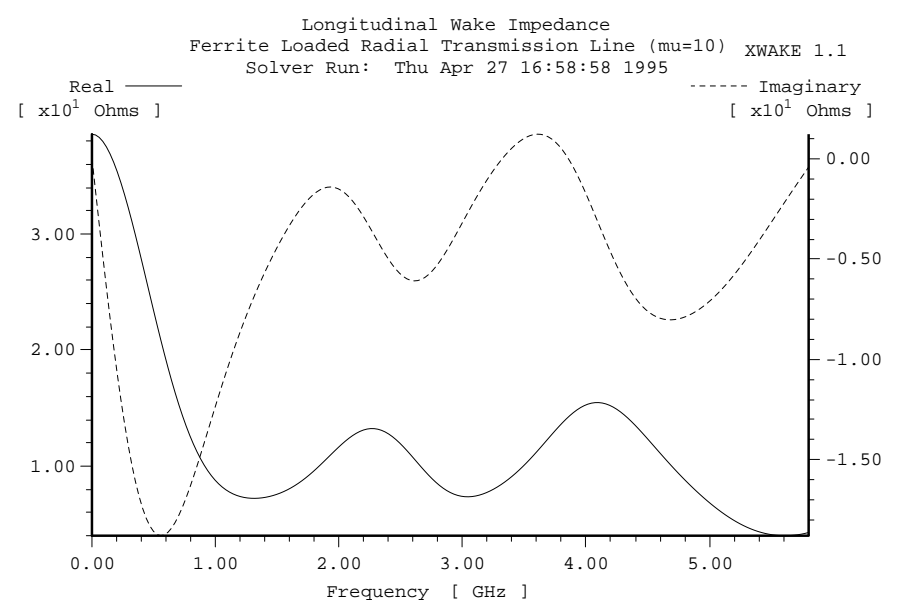

Figure. 6. Monopole Longitudinal Impedance of Ferrite Loaded Radial Transmission Line

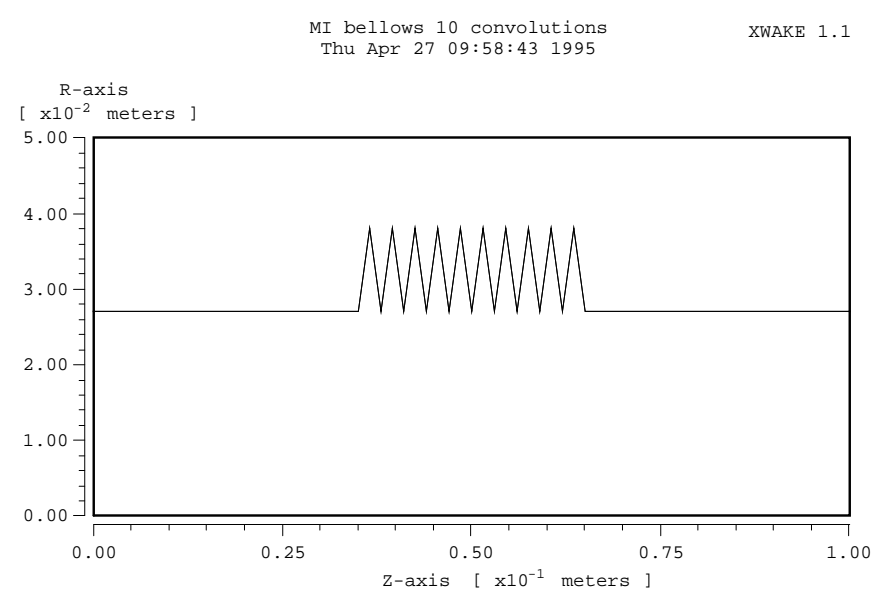

Figure. 7. Bellows Geometry

not converged. The stepped discretization must be reduced to $0.125 \mathrm{~mm}$ for convergence. Hence, contouring provides a saving factor of four in memory and sixteen in time.

\section{SUMMARY}

The salient features of the upcoming 1.1 release of Xwake were discussed. Four features which differentiate Xwake from other codes are: abitrary inhomogeneous material modeling, contour FDTD geometry modeling, the use of a low noise broadband $\mathrm{ABC}$ and implementation of a GUI. Results and validations for both monopole and dipole simulations were presented.

Xwake 1.1 and its users' manual can be obtained via anonymous ftp from Fermilab. For more information contact either author at:

t.jurgens@ieee.org

$$
\text { saewert@crusher.fnal.gov }
$$

\section{References}

[1] T.G. Jurgens and G.W. Saewert, "The Body of Revolution FDTD Algorithm", Chapter 12 in "Computational Electrodynamics: The Finite Difference Time Domain Method", by Allen Taflove, Artech House, 1995.

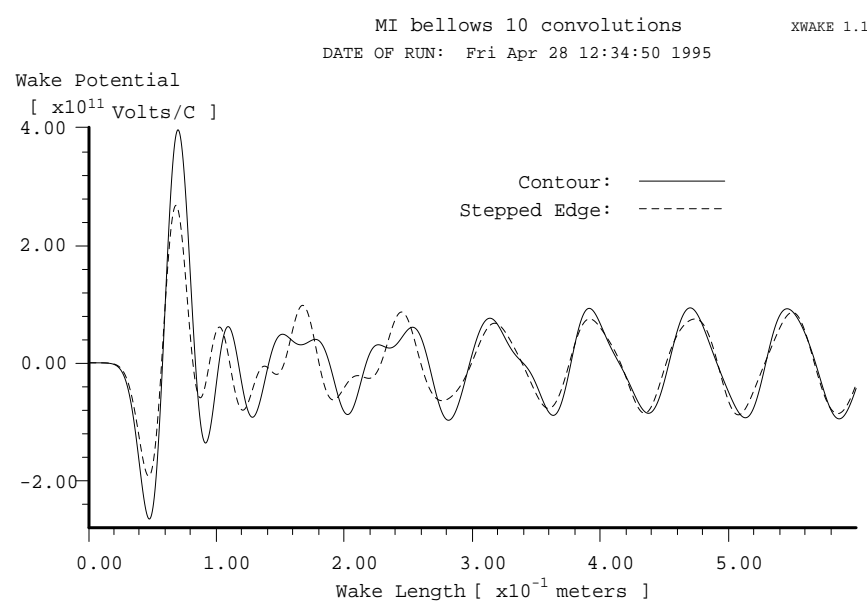

Figure. 8. Bellows Stepped and Contour Wake Comparison

[2] Y. H. Chin, “ABCI User's Guide”, Version 8.8, February 1994.

[3] T. Weiland, "On the Numerical Solution of Maxwell's Equations and Applications in the Field of Accelerator Physics", Particle Accelerators, 15, pp. 245-292, 1984.

[4] T. G. Jurgens and A. Taflove, "Three-Dimensional Contour FDTD Modeling of Scattering from Single and Multiple Bodies”, IEEE Trans. Antennas and Propagat., vol. 41, no. 12, pp. 1703-1708, 1993.

[5] T. G. Jurgens, G. W. Saewert and F. A. Harfoush, "Xwake 1.0: A New Tool for Wakefield and Impedance Calculations", in 1994 European PAC Conference Proceedings, London, UK, May 1994.

[6] T. G. Jurgens, "A Broadband Absorbing Boundary Condition for the FDTD Modeling of Circular Waveguides", 1995 IEEE MTTS Intl. Microwave Symp., Orlando, FL, May 1519. 